\title{
Telehealth for Family Guidance: Acceptance and Commitment Therapy, Parent-Focused Preference Assessment, and Activity-Based Instruction for the Support of Children with Autism Spectrum Disorder and Their Families
}

\author{
Michael J. Cameron ${ }^{1} \cdot$ Tiffanie Moore $^{2} \cdot$ Carla Bogran $^{2} \cdot$ Alexandria Leidt $^{2}$ (D) \\ Published online: 23 June 2020 \\ (C) Association for Behavior Analysis International 2020
}

\begin{abstract}
A 5-stage protocol was used to support Board Certified Behavior Analysts providing telehealth support for children with autism spectrum disorder and their families. Stage 1 of the protocol involves 2 acceptance and commitment therapy exercises. Specifically, the Valued Living Questionnaire is used to identify a family member's values, and the Bull's-Eye Values Survey is employed to determine the extent to which a family member is living in accordance with stated values. Stage 2 of the protocol involves administering an adult version of the Meaningful Activity Participation Assessment to identify preferred activities of parents of a child with autism spectrum disorder. During Stage 3 of the protocol, the goals and objectives of a child with autism spectrum disorder are yoked to the preferred activities of a family member in order to promote child-parent engagement. Stage 4 of the protocol is focused on parent-implemented interventions, and Stage 5 of the protocol is designed as a primer for comprehensive support within an acceptance and commitment therapy model. The 5-stage protocol provides guidelines for Board Certified Behavior Analysts interested in structuring telehealth sessions and optimizing engagement between a family member and a child with autism spectrum disorder.
\end{abstract}

Keywords ACT $\cdot$ Activity-based instruction $\cdot$ Family guidance $\cdot$ Preference assessment $\cdot$ Stage-based intervention

As COVID-19 continues to spread across the United States and face-to-face behavioral health services become more impractical, providers of applied behavior analysis (ABA) services have turned to telehealth to avoid disruption of federally

Editor's Note This manuscript is being published on an expedited basis, as part of a series of emergency publications designed to help practitioners of applied behavior analysis take immediate action to adjust to and mitigate the COVID-19 crisis. This article was submitted on April 20, 2020, and received final acceptance on May 5, 2020. The journal would like to especially thank Dr. Daniel J. Moran for his expeditious review of the manuscript. The views and strategies suggested by the articles in this series do not represent the positions of the Association for Behavior Analysis International or Springer Nature.

Michael J. Cameron

came746@usc.edu

1 Program in Applied Behavior Analysis, University of Southern California, 3620 McClintock Avenue, Los Angeles, CA 90089, USA

2 BlueSprig Pediatrics, Houston, TX, USA mandated coverage for children with autism spectrum disorder (ASD). The recent telehealth initiative has been enabled by commercial- and government-sponsored health insurance companies agreeing to fund telehealth services to support children and families. Telehealth technology is a tool to support the navigation of barriers currently facing $\mathrm{ABA}$ providers; however, substantial challenges (e.g., how to structure a credible telehealth session) need to be overcome for telehealth to achieve its full potential within the field of ABA. In consequence, the development of guidelines and standards for supporting families with a child diagnosed with ASD is an important and valuable process to safeguard the effective and responsible delivery of quality behavior-analytic services.

Families sequestered due to shelter-in-place orders are currently living under conditions involving health risk, economic strain, family and friend separation, psychological vulnerability, and a complete disruption of coveted rituals and routines. Undoubtedly, COVID-19 has altered how families live and what they value. Unexpectedly, for many families, several unpleasant truths have emerged 
during the pandemic: (a) there is uncertainty about how to structure each day for their typically developing children, (b) there is a lack of clarity regarding how to support their child with ASD while faced with insuperable barriers to education and therapy sites, and (c) the discomfort created by economic strain, disrupted living, and a lack of daily structure has put them at risk for collective panic and emotional dysregulation. In this article, we provide a stage-based protocol to support families affected by the COVID-19 pandemic.

An overview of a five-stage protocol to support Board Certified Behavior Analysts (BCBAs) and registered behavior technicians providing telehealth services to families is offered. Specifically, a general overview of five stages of an intervention is provided, including acceptance and commitment therapy (ACT), parent-focused preference assessment, parentimplemented intervention, and parent-led activity-based instruction (ABI). The application of each component of the stage-based model is reviewed. Moreover, following a brief description of each component, guidelines for the implementation of the component are provided. Finally, an overview of a minimum clinical data set to evaluate the efficacy of outcomes is presented.

\section{Acceptance and Commitment Therapy}

According to Harris (2011), ACT is a mindfulness-based therapy used to support behavior change that "challenges the ground rules of most Western psychology. It utilizes an eclectic mix of metaphor, paradox, and mindfulness skills, along with a wide range of experiential exercises and values-guided behavioral interventions" (p. 2). The effectiveness of ACT has been shown in a diverse range of clinical conditions, including depression (Zettle \& Raines, 1989), obsessive-compulsive disorder (Twohig, Hayes, \& Masuda, 2006), workplace stress (Bond \& Bunce, 2000), chronic pain (Dahl, Wilson, \& Nilsson, 2004), and terminal cancer (Branstetter, Wilson, Hildebrandt, \& Mutch, 2004). In addition, pertinent to this article, ACT has been used in the support of parents of children diagnosed with ASD (Blackledge \& Hayes, 2006).

The overall goal of ACT is to promote the requisite psychological flexibility needed to promote human flourishing and welfare. Within ACT, psychological flexibility is achieved by teaching mindfulness skills that enable (a) the acceptance of unwanted thoughts, (b) the preclusion of fusion with unsolicited thoughts and the observation of them without attachment or judgment (i.e., cognitive defusion), (c) the preparedness to be fully aware of the present moment with openness, and (d) the ability to access a preeminent sense of self. Harris (2011) suggested that ACT is a befitting acronym because ACT places emphasis on taking effective action governed by our ineradicable values while we are fully present and engaged. Fundamentally, ACT practitioners use six core principles to support a client's capacity for psychological flexibility: acceptance, defusion, contact with the present moment, the observing self, values, and committed action. Within our stage-based telehealth protocol, we focus our initial attention on values clarification, committed actions, and, ultimately, mindfulness.

\section{Stage 1: Values Clarification and Committed Action}

Values clarification in psychology is hardly a revolutionary concept. The miracle question, for example, has been a chief query guiding constructivist psychology for decades (De Shazer et al., 1986). The miracle question, posed by a psychologist, is designed to evoke a client's specific verbal elaborations centered on the imagined circumstances under which a client's problems are resolved or do not exist (e.g., "If you hear on the news that the shelter-in-place order has been lifted, what would you do tomorrow?'). The process of answering the miracle question results in the identification of what a person truly values. Within ACT, however, there is no attempt to banish, change, or control events. Rather, the goal of ACT is to teach a client how to mitigate the impact of unsolicited thoughts and emotions through the effective use of mindfulness. Nevertheless, as in the values-clarification interventions associated with constructivist psychology, a client's values are determined within ACT, and an array of values-supporting actions are identified.

The first stage within the protocol prioritizes goal clarification and the identification of committed actions. Specifically, Stage 1 of our program begins with two assessments: the Valued Living Questionnaire (VLQ; Wilson, Sandoz, Kitchens, \& Roberts, 2010) and the Bull's Eye Values Survey (BEVS; Lundgren, Luoma, Dahl, Strosahl, \& Melin, 2012). The assessments are used to verify a parent's values and ensure that recommendations provided by a clinician are aligned with a parent's professed values. The assessments are particularly relevant because it is important for BCBAs to avoid values conversion, wherein a family defaults to the values of BCBAs who have earnestly presented their ideas for goals and objectives. The professional literature has shown that adherence to goals is optimized when goals are value focused (Chase et al., 2013).

The VLQ requires respondents (e.g., parents) to answer a series of questions about their values across life domains (e.g., family, marriage, parenting, friendships) and indicate the extent to which they have been living in accordance with these professed values. Areas in which there are large discrepancies between values and the committed actions required to live in consonance with these values are prioritized (e.g., a parent 
may value his or her child's development but struggle to balance priorities to maximize time, attention, and engagement with the child; therefore, actions that support an increased level of attention and time spent with a child are prioritized). Within Stage 1 of the intervention, the VLQ is completed by a parent (privately), and the results are summarized in the context of a telehealth session. Subsequently, the clinician guides each participating parent through the BEVS within the context of a telehealth session. The BEVS is an instrument designed to promote awareness centered on the discrepancy between individuals' values and their actions (i.e., value-action discrepancy). Within the BEVS phase of treatment, a dartboard is projected on a computer screen during a session. According to Lundgren et al. (2012), the simulation of a dartboard is used because most people are acquainted with the idea of tossing darts at a target with concentric rings. The displayed dartboard is partitioned into four regions (e.g., the upper left represents Region 1, and the upper right represents Region 2). Parents are asked to select an area of their lives they value (e.g., family relationships) and to place an $\mathrm{X}$ within one of the multiple rings on the dartboard to indicate the extent to which they are behaving in a manner consistent with their values. One region of the dartboard is used for a single articulated value. An X in the center of the dartboard (i.e., bull's-eye) would signify complete value-action alignment. In contrast, an $\mathrm{X}$ placed within one of the outer rings of the dartboard would visually reveal a discrepancy between values and actions. Within the BEVS, four values are identified as a result of the VLQ. Subsequently, the BEVS allows for a value-action cohesiveness assessment. Areas of value-action discrepancy are prioritized for intervention. The intention of Stage 1 is to identify a parent's values, detect discrepancies between stated values and committed actions, and then connect a child's need for instruction to the parent's declared values. For example, if a family member indicates he or she values creative expression, but just does not find the time to do anything of a creative nature, then a child's instructional needs (e.g., fine motor development) would be connected to a specific activity the family member valued. Specific activities are identified as a result of a parent-focused preference assessment.

\section{Stage 2: Parent-Focused Preference Assessment}

Preference assessments have been traditionally used to identify putative reinforcers for an individual with ASD. Researchers have shown that individuals with ASD and related disabilities can demonstrate, through a variety of preference assessment formats, clear preferences for activities and items that are a source of visual, olfactory, gustatory, thermal, social, vestibular, auditory, or tactile stimulation (Mason, McGee, Farmer-Dougan, \& Risley, 1989). Research studies have shown that when preference assessments are integrated into programs for addressing both behavioral deficits and behavioral excesses, an individual's ability to choose activities and items of high interest results in skill acquisition, increases in appropriate behavior, and quality-of-life changes. Although there is clear evidence supporting the use of preference assessments to identify putative reinforcers for individuals with ASD and related disabilities, such choice-based interventions have been used less often within behavior-analytic treatment to identify the activity preferences of family members (e.g., parents). The identification of the activity preferences of family members is essential for several reasons. First, if activities preferred by a parent of a child with ASD can be identified, those activities can then be used as the basis for engagement with that child. Next, with the support of a BCBA, detailed suggestions centered on how a child's goals and objectives can be addressed, within the context of a parent's preferred activities, can be offered. For example, a mother who enjoys woodworking could use the hobby to address her child's goals for gross motor skill development (e.g., marching across the room to get a piece of wood), fine motor skill development (e.g., painting a wooden surface), and language and communication skill development (e.g., manding for information or the opportunity to conjoin two pieces of wood, such as a dovetail and joint). Finally, a parent's preferred activities can serve as the basis for increasing the number of learning opportunities a child receives and potentially improve the likelihood of acquisition of socially significant skills.

Although preference assessments for adults without disabilities are not discussed in journals devoted to behavior analysis, they are described in other disciplines. In fact, within contemporary theories of adult development, theorists have linked meaningful life engagement to a sense of well-being. In consequence, instruments such as the Meaningful Activity Participation Assessment (MAPA) have been identified to assess activities that are personally valued and meaningful to adults (Eakman, Carlson, \& Clark, 2010). Therefore, within Stage 2 of our protocol, an activity preference assessment is conducted with participating parents. Specifically, an indirect preference assessment is coordinated within the context of a telehealth session. To facilitate the preference assessment, MAPA is used as an inspiration for the construction of a family-centered and customized tool. Within Stage 2, a checklist-driven survey is used. The telehealth practitioner conducts parent interviews to assess the frequency of activity participation, and the degree of personal meaningfulness experienced with each activity. Next, the telehealth BCBA links the results of the VLQ and the BEVS with the outcomes of the customized assessment. Subsequently, a list of activities that address parents' values, and reconcile with their preferred activities, is created. For example, if the BCBA learns that physical self-care (e.g., exercise) is valued by a family member (as indicated in the VLQ assessment), but there is a relevant 
discrepancy between the family member's value for self-care and her or his committed actions (as verified in the BEVS results), and the family member selects physical exercise as a preference from the parent-centered assessment, then a list of activities involving physical exercise is generated. All activities suggested, however, must be suitable for a child's inclusion.

\section{Stage 3: Goal Alignment and Parent-Led Activity-Based Intervention}

Within Stage 3 of the intervention, the telehealth practitioner uses a visual framework that lists (a) the parent's values (as identified with the BEVS), (b) the parent's preferred activities (as revealed with the BEVS), (c) the materials required to support those activities, (d) the child's treatment goals, (e) examples of how the child's treatment goals could be addressed within the context of each preferred activity, (f) the time of day activities could occur, $(\mathrm{g})$ the preferred duration and frequency of the activity (selected by the parent), (h) the type of data that could be collected to capture the child's performance, (i) recommendations for the generalization of skills, and (j) the desired outcomes. The documented framework is prepared in advance of a parent meeting and reviewed in a telehealth session (see Table 1).

Subsequently, a participating parent is taught a variation of ABI. Traditionally, ABI involves a child-directed transactional approach that integrates a child's individualized goals and objectives in a routine or within planned or child-initiated activities. The approach capitalizes on naturally occurring antecedents and consequences to develop functional and generative skills (Bricker \& Cripe, 1992). Within Stage 3 of our protocol, we develop a parentdirected transactional approach that yokes a child's individualized goals and objectives to a planned and parentled activity. The intervention is then implemented within Stage 4 of the protocol.

\section{Stage 4: Parent-Implemented Intervention and Data Collection}

Researchers have demonstrated that parents can acquire and apply evidence-based strategies with which to teach their children (Roberts \& Kaiser, 2011). In fact, parent-implemented intervention is an evidence-based practice (Wong et al., 2015). However, parents require the support of high-quality guidance and coaching to ensure that programs are implemented with fidelity. Within Stage 4 of the protocol, a telehealth-based behavioral skills training (BST) model is used that includes (a) the BCBA providing verbal and written instructions to support parent-implemented ABI, (b) the BCBA modeling ABI with a child from the distance site (i.e., where the BCBA is located), (c) the parent role-playing ABI from the originating site (i.e., where the parent is located), and (d) the BCBA providing feedback to the parent. BST has been shown to be an effective method for supporting parents in the implementation of behavioral interventions (Drifke, Tiger, \& Wierzba, 2017). Permanent-product data are collected by way of the video messaging app Marco Polo, a free video and walkie-talkie app that allows end users (i.e., the BCBA and parent) to quickly send short video-based messages back and forth during a session. The Marco Polo app is used (a) to

Table 1. Goal Alignment and Parent-Led ABI

\begin{tabular}{|c|c|c|}
\hline Element & Description & Example \\
\hline Parent interest & $\begin{array}{l}\text { Based on the Valued Living Questionnaire and the Bull's-Eye } \\
\text { Values Survey, identify the caregiver's values. Conduct an } \\
\text { activity preference assessment with the parent using the } \\
\text { Meaningful Activity Participation Assessment. }\end{array}$ & $\begin{array}{l}\text { Mom identified family as a value and gardening as a preferred } \\
\text { activity. The family has a garden in the backyard of the home } \\
\text { and both adult- and child-sized gardening tools in the shed. }\end{array}$ \\
\hline Treatment goal & $\begin{array}{l}\text { Select a treatment goal from the child's program that aligns with the } \\
\text { parent's values and activities of interest. }\end{array}$ & $\begin{array}{l}\text { Manding: requesting items necessary to complete tasks using } \\
\text { four- to five-word sentences. } \\
\text { The child can request items needed to participate in gardening. }\end{array}$ \\
\hline Time of day & Select the time of day and duration for the selected activity. & $\begin{array}{l}\text { Mom gardens before lunch every day. } \\
\text { The child can attend to activities for approximately } 10-15 \mathrm{~min} \text { at } \\
\text { a time. }\end{array}$ \\
\hline Data collection & Collect the child's language samples. & $\begin{array}{l}\text { Use Marco Polo }{ }^{\mathrm{TM}} \text { to capture permanent-product data on the } \\
\text { language used during the gardening activity. }\end{array}$ \\
\hline Generalization & Four- to five-word mands are demonstrated in novel contexts. & $\begin{array}{l}\text { The child demonstrates the ability to ask for items using four- to } \\
\text { five-word mands in other activities (e.g., cooking, playing with } \\
\text { a sibling). }\end{array}$ \\
\hline Outcomes & Four- to five-word mands are used spontaneously in novel contexts. & $\begin{array}{l}\text { The child spontaneously uses four- to five-word mands in novel } \\
\text { contexts (e.g., with extended family members, during } \\
\text { mealtimes). }\end{array}$ \\
\hline
\end{tabular}


promote engagement between the BCBA and parent, (b) to capture videos of a child's skills for the purpose of data collection, and (c) to create videos that can be used as a source of feedback to the child and shown to other family members to reveal the capabilities of a child (i.e., the Marco Polo app has a save and forward feature that allows family members to share brief videos). Within Stage 4 of the protocol, participating parents implement $\mathrm{ABI}$ and take responsibility for data collection via the Marco Polo app.

\section{Stage 5: Priming for a Comprehensive Model of ACT}

The transtheoretical model (TTM) provides a framework for both the conceptualization and measurement of behavior change (Prochaska \& Velicer, 1997). The TTM was developed as a comprehensive model of behavior change. The model incorporates cognitive, behavioral, and temporal aspects into a unified approach for behavior change. The TTM facilitates the promotion of strategies that are stage matched, individualized, and easily adapted. Prochaska and Velicer (1997) outlined five stages in their behavior-change model: (a) a precontemplation stage wherein a person is inactive; (b) a contemplation stage characterized by a person's willingness to discuss future plans for changing behavior; (c) a preparation stage in which a person begins the process of establishing process goals, short-term goals, and long-term goals for behavior change; (d) an action stage characterized by a person's full engagement in the behavior-change process; and lastly, (e) a maintenance stage, defined by a person's focus on relapse prevention and the maintenance of newly acquired behaviors. Prochaska and Velicer (1997) emphasized the importance of using "stage-matched" interventions. More to the point, any intervention proposed for an individual should be commensurate with his or her current level of motivation. For example, trying to persuade a person in a precontemplation stage (i.e., inactive) to immediately initiate some form of behavior change is not advised (e.g., bringing a friend, who enjoys the consolations of the couch, for a surprise visit to the yoga studio is misguided). An appropriate stage-matched intervention for a person in a precontemplation or contemplation stage of behavior change could involve exposing a person to educational material. In consequence, within Stage 5 of our protocol, we use an educational approach to prepare a family member to use a comprehensive model of ACT. Orienting a person to educational information facilitates a smoother downstream flow with a person preparing for transition and change. A matrix was developed to provide a visual representation of exercises to support each of the six core principles because the six core principles in ACT are associated with a series of exercises to allow for ongoing practice (i.e., acceptance, defusion, contact with the present moment, the observing self, values, and committed action). For example, in order to practice the skill of resisting attachment to unwanted thoughts and emotions, 10 exercises were outlined, each with a brief description: (a) leaves on a stream, (b) hands in front of your face, (c) the guest house, (d) playground bully, (e) silly voice, (f) the view from above, (g) Plato's IMAX, (h) the sky and the clouds, (i) flushed with pride, and (j) you are the Buddha (see Table 2).

Subsequently, a choice intervention is used, wherein the parent is given the autonomy to select the exercise to be explored. Next, we use a behavioral rehearsal strategy (Beidas, Cross, \& Dorsey, 2014). Behavioral rehearsal involves engaging the parent in a simulated interaction with the BCBA; however, the parent assumes the instructor's role, and the BCBA welcomes the role of the trainee. The parent is asked to select at least one exercise from a preferred category for a behavioral rehearsal session (i.e., acceptance, defusion, contact with the present moment, the observing self, values, and committed action). All behavioral rehearsals occur in the context of a telehealth session. The behavioral rehearsal strategy allows a family member to experience at least one exercise associated with each of the core principles of ACT. The behavioral rehearsal approach agrees with Prochaska and Velicer's (1997) stage-matched intervention to support behavior change. Moreover, the behavioral rehearsal strategy allows the $\mathrm{BCBA}$ to ensure that the parent experiences the instructional format as judicious and nonjudgmental. In our opinion, it is critical for a BCBA to identify a family member's current state with respect to his or her own behavior-change process (e.g., precontemplation, contemplation, preparation, action, maintenance) before imposing expectations involving change. Our stage-based intervention concludes with an outline of a minimum clinical data set influenced by frameworks for accountability within health care.

\section{A Minimum Clinical Data Set for Telehealth}

Using Berwick's (2002) framework of quality, we created a minimum clinical data set for the evaluation of our five-stage intervention model. According to Berwick, quality can be achieved by addressing processes at four levels: that of the client, that of the microsystem, that of the organization, and that of the larger physical, social, economic, and political environment. At the client level (i.e., the child receiving services), our data collection system includes pre- and postmeasures centered on (a) the number of programs per domain (e.g., language, motor skills) implemented by a family member, (b) the number of learning opportunities the child receives per domain, (c) the duration of time spent (with a 
Table 2. Ten Defusion Exercises

\begin{tabular}{|c|c|}
\hline Element & Description \\
\hline Leaves on a stream & As thoughts pass by, write them on leaves and drop them into the stream in front of you to watch them float away. \\
\hline Hands in front of your face & Cover your face with your hands, and slowly move your hands away from your face. \\
\hline The guest house & Greet different thoughts, feelings, and versions of yourself as if they were guests in a house. \\
\hline Playground bully & Treat your negative thoughts and feelings the same way you would a school or playground bully. \\
\hline Silly voice & Write down any negative thoughts and feelings, then say them out loud in a silly voice (e.g., cartoon character). \\
\hline The view from above & $\begin{array}{l}\text { Think of your situation and life, then visualize zooming out from your life until you are zoomed all the way out to the solar } \\
\text { system or galaxy. }\end{array}$ \\
\hline Plato's IMAX & $\begin{array}{l}\text { Imagine your thoughts and feelings as you would see them on a movie screen with yourself sitting in the audience, } \\
\text { watching. }\end{array}$ \\
\hline The sky and the clouds & Look up to the sky, and watch the clouds pass by. The mind is the sky, and the clouds are thoughts and feelings. \\
\hline Flushed with pride & $\begin{array}{l}\text { Write down the negative thought or feeling, fold it up, and flush the paper down the toilet. Thank the thought or feeling for } \\
\text { helping, and note you are now letting it go the same as you let go of food after taking nutrition from it. }\end{array}$ \\
\hline You are the Buddha & $\begin{array}{l}\text { Imagine sitting in front of the Buddha. Note the positive thoughts and feelings. Imagine integrating with the Buddha and } \\
\text { recognizing the Buddha within you (e.g., the highest version of yourself). }\end{array}$ \\
\hline
\end{tabular}

family member) engaged in ABI, (d) the number of skills mastered per domain, and (e) the integration of acquired skills into the child's daily routine. At the level of the microsystem (i.e., the family), our pre- and postintervention assessment protocol includes the administration of the Family Adaptability and Cohesion Evaluation Scale (Olson, Portner, $\&$ Bell, 1982). It is our position that a goodness of fit between interventions and outcome measures is necessary. An assessment tool designed to evaluate changes in flexibility and adaptability is apropos because the primary goal of ACT is to increase psychological flexibility. Adaptability, within the structure of a nuclear family, is the ability of family members to change their power structure, role relationships, and rules to respond to situational and developmental changes. Therefore, an assessment tool designed to measure flexibility and adaptability is required. In addition, the Parental Locus of Control Scale (Lloyd \& Hastings, 2009) and a social validity measure are also part of the full-battery assessment.

From an organizational standpoint, the COVID-19 pandemic has necessitated the redesign of organizational processes, moving from traditional models of service delivery (e.g., center-based services) toward client-centered structures involving the use of telehealth. This change has caused ABA providers to engage in a redesign process that encompasses several restructuring actions, including (a) changing the form of service delivery (i.e., from direct and in-person treatment to telehealth services), (b) changing staff training protocols (e.g., methods for developing relationships and building rapport via telecommunications), and (c) changing methods for managing new client intake, assessment, and treatment planning. We identified two measures to evaluate the adaptability and agility of an organization. First, we created a dashboard to generate utilization data (i.e., the percentage of service hours delivered with respect to the total number of hours authorized by the payer) to evaluate the organization's commitment to the continuity of service. Second, we developed an employee satisfaction survey to measure clinicians' perceived preparedness for functioning within a telehealth environment. Finally, to maintain a framework of accountability to the organization's larger regulatory and political environment (i.e., the payers), we developed an internal auditing system to ensure that errorfree claims were submitted. All measures were generated to comprehensively evaluate the efficacy and practicality of utilizing the stage-based model for delivering telehealth services to children with ASD and their families.

\section{Conclusion}

The protocol we developed is about embracing the rules but not the status quo. We describe a stage-based model for providing telehealth services to children with ASD and their families. The stage-based model comprises a synthesis of information from contextual behavioral science, ABA, health psychology, and occupational therapy. Specifically, the stagebased model includes ACT, a parent-focused preference assessment inspired by work in the field of occupational therapy, and parent-led ABI. The process concludes with the outline of a minimum clinical data set influenced by extant initiatives in health care. At this juncture, the efficacy of the fivestage model requires further evaluation. In conclusion, as written by Hayes, Barnes-Holmes, and Wilson (2012), "as a functional contextualist sees it, the ultimate purpose of behavioral science is to change the world in a positive and intentional way" (p. 2). We do not nurture any desire for the field of ABA to continue its trajectory prior to the pandemic; rather, we view the changes in daily practices brought about by the COVID-19 pandemic as a catalyst for innovation in the field of ABA. 


\section{Compliance with Ethical Standards}

Conflict of interest The authors declare that they have no conflict of interest.

\section{References}

Beidas, R. S., Cross, W., \& Dorsey, S. (2014). Show me, don't tell me: Behavioral rehearsal as a training and analogue fidelity tool. Cognitive and Behavioral Practice, 21(1), 1-11. https://doi.org/10. 1016/j.cbpra.2013.04.002.

Berwick, D. M. (2002). A user's manual for the IOM's "Quality Chasm" report. Health Affairs, 21, 80-90. https://doi.org/10.1377/hlthaff.21. 3.80 .

Blackledge, J. T., \& Hayes, S. C. (2006). Using acceptance and commitment training in the support of parents of children diagnosed with autism. Child \& Family Behavior Therapy, 28(1), 1-18. https://doi. org/10.1300/J019v28n01_01.

Bond, F. W., \& Bunce, D. (2000). Mediators of change in emotionfocused and problem focused worksite stress management interventions. Journal of Occupational Health Psychology, 5, 156-163. https://doi.org/10.1037/1076-8998.5.1.156.

Branstetter, A. D., Wilson, K. G., Hildebrandt, M., \& Mutch, D. (2004). Improving psychological adjustment among cancer patients: $A C T$ and CBT. Paper presented at the Association for Advancement of Behavior Therapy. Louisiana: New Orleans.

Bricker, D., \& Cripe, J. (1992). An activity-based approach to early intervention. Baltimore, MD: Brookes. https://doi.org/10.1097/ 00001577-199200440-00023.

Chase, J. A., Houmanfar, R., Hayes, S. C., Ward, T. A., Vilardaga, J. P., \& Folletee, V. (2013). Values are not just goals: Online ACT-based values training adds to goal setting in improving undergraduate college student performance. Journal of Contextual Behavioral Science, 2(3-4), 79-84. https://doi.org/10.1016/j.jcbs.2013.08.002.

Dahl, J., Wilson, K. G., \& Nilsson, A. (2004). Acceptance and commitment therapy and the treatment of persons at risk for long-term disability resulting from stress and pain symptoms: A preliminary randomized trial. Behavior Therapy, 35, 785-802. https://doi.org/ 10.1016/S0005-7894(04)80020-0.

De Shazer, S., Berg, I. K., Lipchik, E., Nunnaly, E., Molnar, A., Gingerich, W., \& Weiner-Davis, M. (1986). Brief therapy: Focused solution development. Family Process, 25(2), 207-221. https://doi.org/10.1111/j.1545-5300.1986.00207.x.

Drifke, M. A., Tiger, J. H., \& Wierzba, B. (2017). Using behavioral skills training to teach parents to implement three-step prompting: A component analysis and generalization assessment. Learning and Motivation, 57, 1-14. https://doi.org/10.1016/j.lmot.2016.12.001.

Eakman, A. M., Carlson, M. E., \& Clark, F. A. (2010). The Meaningful Activity Participation Assessment: A measure of engagement in per- sonally valued activities. International Journal of Aging and Human Development, 70(4), 299-317. https://doi.org/10.2190/AG.70.4.b.

Harris, R. (2011). Embracing your demons: An overview of acceptance and commitment therapy. Retrieved from https://www. psychotherapy.net/article/Acceptance-and-Commitment-TherapyACT

Hayes, S. C., Barnes-Holmes, D., \& Wilson, K. G. (2012). Contextual behavioral science: Creating a science more adequate to the challenge of the human condition. Journal of Contextual Behavioral Science, 1(1-2), 1-16. https://doi.org/10.1016/j.jcbs.2012.09.004.

Lloyd, T., \& Hastings, R. P. (2009). Parental locus of control and psychological well-being in mothers of children with intellectual disability. Journal of Intellectual \& Developmental Disability, 34(2), 104-115. https://doi.org/10.1080/13668250902862074.

Lundgren, T., Luoma, J., Dahl, J., Strosahl, K., \& Melin, L. (2012). The Bull's-Eye Values Survey: A psychometric evaluation. Cognitive and Behavioral Practice, 19(4), 518-526. https://doi.org/10.1016/ j.cbpra.2012.01.004.

Mason, S. A., McGee, G. G., Farmer-Dougan, V., \& Risley, T. R. (1989). A practical strategy for ongoing reinforcer assessment. Journal of Applied Behavior Analysis, 22(2), 171-179. https://doi.org/10.1901/ jaba.1989.22-171.

Olson, D. H., Portner, J., \& Bell, R. Q. (1982). FACES II: Family Adaptability and Cohesion Evaluation Scales. Minneapolis, MN: Family Social Science, University of Minnesota.

Prochaska, J. O., \& Velicer, W. F. (1997). The transtheoretical model of health behavior change. American Journal of Health Promotion, 12(1), 38-48. https://doi.org/10.4278/0890-1171-12.1.38.

Roberts, M. Y., \& Kaiser, A. P. (2011). The effectiveness of parentimplemented language interventions: A meta-analysis. American Journal of Speech-Language Pathology, 20, 180-199. https://doi. org/10.1044/1058-0360(2011/10-0055).

Twohig, M. P., Hayes, S. C., \& Masuda, A. (2006). Increasing willingness to experience obsessions: Acceptance and commitment therapy as a treatment for obsessive compulsive disorder. Behavior Therapy, 37(1), 3-13. https://doi.org/10.1016/j.beth.2005.02.001.

Wilson, K. G., Sandoz, E. K., Kitchens, J., \& Roberts, M. (2010). The Valued Living Questionnaire: Defining and measuring valued action within a behavioral framework. The Psychological Record, 60, 249-272. https://doi.org/10.1007/BF03395706.

Wong, C., Odom, S. L., Hume, K. A., Cox, W. A., Fettig, A., Kucharczyk, S., et al. (2015). Evidence-based practices for children, youth, and young adults with autism spectrum disorder: A comprehensive review. Journal of Autism and Developmental Disorders, 45, 1951-1966. https://doi.org/10.1007/s10803-014-2351-z.

Zettle, R. D., \& Raines, J. C. (1989). Group cognitive and contextual therapies in treatment of depression. Journal of Clinical Psychology, 45, 438-445. https://doi.org/10.1002/10974679(198905)45:3<436::AID-JCLP2270450314>3.0.CO;2-L.

Publisher's Note Springer Nature remains neutral with regard to jurisdictional claims in published maps and institutional affiliations. 